\title{
THE SIMPLEST RANDOM WALKS FOR THE DIRICHLET PROBLEM*
}

\author{
G. N. MILSTEIN ${ }^{\dagger}$ AND M. V. TRETYAKOV $\ddagger$
}

(Translated by the authors)

\begin{abstract}
The Dirichlet problem for both parabolic and elliptic equations is considered. A solution of the corresponding characteristic system of stochastic differential equations is approximated in the weak sense by a Markov chain. If a state of the chain comes close to the boundary of the domain in which the problem is considered, then in the next step the chain either stops on the boundary or goes inside the domain with some probability due to an interpolation law. An approximate solution of the Dirichlet problem has the form of expectation of a functional of the chain trajectory. This makes it possible to use the Monte Carlo technique. The proposed methods are the simplest ones because they are based on the weak Euler approximation and linear interpolation. Convergence theorems, which give accuracy orders of the methods, are proved. Results of some numerical tests are presented.
\end{abstract}

Key words. Dirichlet problem for parabolic and elliptic equations, probabilistic representations, weak approximation of solutions of stochastic differential equations, Markov chains, random walks

PII. S0040585X97979433

1. Introduction. Let $G$ be a bounded domain in $\mathbf{R}^{d}$ and $Q=G \times\left[T_{0}, T\right)$ be a cylinder in $\mathbf{R}^{d+1}, \Gamma=\bar{Q} \backslash Q$ be the part of the cylinder boundary consisting of the upper base and lateral surface. Consider the Dirichlet problem for the parabolic equation

$$
\begin{gathered}
\frac{\partial u}{\partial t}+\frac{1}{2} \sum_{i, j=1}^{d} a^{i j}(t, x) \frac{\partial^{2} u}{\partial x^{i} \partial x^{j}}+\sum_{i=1}^{d} b^{i}(t, x) \frac{\partial u}{\partial x^{i}} \\
+c(t, x) u+g(t, x)=0, \quad(t, x) \in Q, \\
\left.u\right|_{\Gamma}=\varphi(t, x) .
\end{gathered}
$$

The form of (1.1) is convenient for a probabilistic approach: the "initial" condition is prescribed at the final time moment $t=T$ and the equation is considered for $t<T$.

We assume that the coefficients $a^{i j}=a^{j i}$ satisfy the strict ellipticity condition in $\bar{Q}$ and that conditions hold which guarantee existence of the classical solution $u(t, x)$ of the problem (1.1)-(1.2) having continuous in $\bar{Q}$ derivatives

$$
\frac{\partial^{2} u}{\partial t^{2}}, \quad \frac{\partial^{3} u}{\partial t \partial x^{i} \partial x^{j}}, \quad \frac{\partial^{3} u}{\partial x^{i} \partial x^{j} \partial x^{k}}, \quad \frac{\partial^{4} u}{\partial x^{i} \partial x^{j} \partial x^{k} \partial x^{l}} .
$$

*Received by the editors November 16, 1999. This work was partially supported by Russian Foundation for Basic Research grant 99-01-00134.

http://www.siam.org/journals/tvp/47-1/97943.html

${ }^{\dagger}$ Department of Mathematics, Ural State University, Lenin St. 51, 620083 Ekaterinburg, Russia. Current address: Weierstraß-Institut für Angewandte Analysis und Stochastik, Mohrenstr. 39, D10117 Berlin, Germany (Grigori.Milstein@usu.ru, Milstein@wias-berlin.de).

${ }^{\ddagger}$ Institute of Mathematics and Mechanics, Russian Academy of Sciences, S. Kovalevskaya St. 16, 620219 Ekaterinburg, Russia. Current address: Department of Mathematics and Computer Science, University of Leicester, Leicester LE1 7RH, UK (Michael.Tretyakov@usu.ru, M.Tretiakov@le.ac.uk). 
It is sufficient for the above to require [1] that the functions $a^{i j}(t, x), b^{i}(t, x), c(t, x)$, $g(t, x)$, together with their first partial derivatives in $t, x^{k}$ and second partial derivatives in $x^{k}$, be continuous in $\bar{Q}$; the domain $G$ have a twice continuously differentiable boundary $\partial G$; the function $\varphi(t, x),(t, x) \in \Gamma$, be at least of the same smoothness as the solution $u(t, x)$; and, finally, that the function $\varphi(t, x)$ satisfy (1.1) on the boundary of the upper base of the cylinder $\bar{Q}$.

The solution of problem (1.1)-(1.2) has the following probabilistic representation:

$$
u(t, x)=\mathbf{E}\left[\varphi\left(\tau, X_{t, x}(\tau)\right) Y_{t, x, 1}(\tau)+Z_{t, x, 1,0}(\tau)\right]
$$

where $X_{t, x}(s), Y_{t, x, y}(s), Z_{t, x, y, z}(s), s \geqq t$, is the solution of the Cauchy problem for the system of stochastic differential equations (SDEs)

$$
\begin{aligned}
d X & =(b(s, X)-\sigma(s, X) \mu(s, X)) d s+\sigma(s, X) d w(s), & X(t) & =x, \\
d Y & =c(s, X) Y d s+\mu^{\mathrm{T}}(s, X) Y d w(s), & Y(t) & =y, \\
d Z & =g(s, X) Y d s+F^{\mathrm{T}}(s, X) Y d w(s), & Z(t) & =z,
\end{aligned}
$$

$(t, x) \in Q$, and $\tau=\tau_{t, x}$ is the first exit time of the trajectory $\left(s, X_{t, x}(s)\right)$ to the boundary $\Gamma$. In (1.4)-(1.6), w(s) $=\left(w^{1}(s), \ldots, w^{d}(s)\right)^{\top}$ is a standard Wiener process, $b(s, x)$ is a $d$-dimensional column-vector composed of the coefficients $b^{i}(s, x)$, the $d \times d$ matrix $\sigma(s, x)$ is obtained from the formula

$$
\sigma(s, x) \sigma^{\mathrm{T}}(s, x)=a(s, x), \quad a(s, x)=\left\{a^{i j}(s, x)\right\}, \quad i, j=1, \ldots, d,
$$

$\mu(s, x)$ and $F(s, x)$ are arbitrary $d$-dimensional column-vectors sufficiently smooth in $\bar{Q}, Y$, and $Z$ are scalars.

For $\mu(s, x)=0$ and $F(s, x)=0$, formula (1.3) gives the standard probabilistic representation (see [2] and [3]). For $\mu(s, x) \neq 0$ and $F(s, x)=0$, (1.3) follows from Girsanov's theorem. It is also clear that the term $F(s, x) \neq 0$ does not affect the validity of this formula. Thus, the mean of the random variable appearing under the symbol of expectation in (1.3) does not depend on $\mu$ and $F$. At the same time, other properties of this random variable can essentially depend on $\mu$ and $F$. In particular, choosing $\mu$ (for $F=0$ ), it is possible to reach zero variance of this random variable [4]. The same result can be achieved by choosing $F$ (for $\mu=0$ ) [5]. This property is of great importance since we use the Monte Carlo technique for solving linear boundary value problems by probabilistic methods. This is a reason for us to construct random walks taking into account dependence on $\mu$ and $F$.

Difficulties arising in realizing the probabilistic representation (1.3) were discussed in [6]. For instance, one of the difficulties consists of the following. The difference $\tau-t$ in (1.3) can take arbitrary small values and, consequently, it is impossible to integrate numerically system (1.4) with a fixed time step. In particular, we cannot use meansquare Euler approximations. Of course, dealing with realization of the expectation in (1.3), it is natural to exploit more simple, weak approximations, imposing on them some restrictions related to nonexit from the domain $\bar{Q}$. Such approximations were constructed in [6]. One of the main ideas consists of controlling the time step of numerical integration of system (1.4): the step is chosen so that (of course, aside from reaching a required accuracy) the next state of a Markov chain approximating, in the weak sense, the solution of (1.4) remains in the domain $\bar{Q}$ with probability one. This leads to a decrease of the time step when the chain is close to the boundary $\Gamma$ of the 
domain $Q$. The chain is stopped in a narrow zone near the boundary so that values of the solution $u(t, x)$ in this zone can be approximated quite accurately by the known values of the function $\varphi$ on the boundary.

In this paper, the step of numerical integration of system (1.4) is constant for points belonging to a certain time layer $t=t_{k}$. But when a point is close to the boundary, we make an intermediate (auxiliary) step of the random walk, which preserves the point in the time layer $t=t_{k}$. The result of this auxiliary step is such that the point, which is close to the boundary, is replaced by two points with some probabilities using an interpolation. One of these new points belongs to the boundary, and, if it is realized, the random walk terminates. The other point is inside the domain so that starting from it we can make a new step of numerical integration without leaving the domain $\bar{Q}$. The approach developed in [6] is probably more universal. However, the methods proposed in this paper are of independent interest from both theoretical and applied points of view due to their simplicity.

The main algorithm of random walk is proposed in the next section. In section 3, its convergence with weak order of accuracy $O(h)$ is proved. Some similar algorithms of random walks are given in section 4 . One of these algorithms is distinguished by being the simplest but its order of convergence is $O(\sqrt{h})$. In section 5 , the results of the previous sections are carried over to the Dirichlet problem for elliptic equations. Finally, some numerical tests of the proposed methods are presented in section 6 .

2. The algorithm of random walk. We apply the weak explicit Euler approximation with the simplest simulation of noise to system (1.4)-(1.6):

$$
\begin{aligned}
X_{t, x}(t+h) & \approx X=x+h(b(t, x)-\sigma(t, x) \mu(t, x))+h^{1 / 2} \sigma(t, x) \xi, \\
Y_{t, x, y}(t+h) & \approx Y=y+h c(t, x) y+h^{1 / 2} \mu^{\mathrm{T}}(t, x) y \xi \\
Z_{t, x, y, z}(t+h) & \approx Z=z+h g(t, x) y+h^{1 / 2} F^{\mathrm{T}}(t, x) y \xi
\end{aligned}
$$

where $h>0$ is a step of integration (a sufficiently small number), and $\xi=\left(\xi^{1}, \ldots, \xi^{d}\right)^{\top}$, $\xi^{i}, i=1, \ldots, d$, are mutually independent random variables taking the values \pm 1 with probability $\frac{1}{2}$. Clearly, the random vector $X$ takes $2^{d}$ different values.

Introduce the set of boundary points (a boundary zone) $S_{t, h} \subset \bar{G}$ on the layer $t$ : we say that $x \in S_{t, h}$ if at least one of the $2^{d}$ values of the vector $X$ is outside $\bar{G}$. It is not difficult to see that due to compactness of $\bar{Q}$ there is a constant $\lambda>0$ such that if the distance from $x$ to the boundary $\partial G$ is equal to or greater than $\lambda \sqrt{h}$, then $x$ is not a boundary point and, therefore, for such $x$ all the realizations of the random variable $X$ belong to $\bar{G}$.

Since restrictions connected with nonexit from the domain $\bar{G}$ should be imposed on an approximation of system (1.4), formulas (2.1)-(2.3) can be used only for the points $x \in \bar{G} \backslash S_{t, h}$ on the layer $t$, and a special construction is required for boundary points.

Let $x \in S_{t, h}$. Denote by $x^{\pi} \in \partial G$ the projection of the point $x$ on the boundary of the domain $G$ (the projection is unique since $h$ is sufficiently small and $\partial G$ is smooth) and by $n\left(x^{\pi}\right)$ the unit vector of internal normal to $\partial G$ at $x^{\pi}$. Introduce the random vector $X_{x, h}^{\pi}$ taking two values $x^{\pi}$ and $x+h^{1 / 2} \lambda n\left(x^{\pi}\right)$ with probabilities $p=p_{x, h}$ and $q=q_{x, h}=1-p_{x, h}$, respectively, where

$$
p_{x, h}=\frac{h^{1 / 2} \lambda}{\left|x+h^{1 / 2} \lambda n\left(x^{\pi}\right)-x^{\pi}\right|} .
$$


If $v(x)$ is a twice continuously differentiable function with the domain of definition $\bar{G}$, then an approximation of $v(x)$ by the expectation $\mathbf{E} v\left(X_{x, h}^{\pi}\right)$ corresponds to linear interpolation and

$$
v(x)=\mathbf{E} v\left(X_{x, h}^{\pi}\right)+O(h)=p v\left(x^{\pi}\right)+q v\left(x+h^{1 / 2} \lambda n\left(x^{\pi}\right)\right)+O(h) .
$$

We emphasize that the second value $x+h^{1 / 2} \lambda n\left(x^{\pi}\right)$ is not a boundary point. We also note that $p$ is always greater than $\frac{1}{2}$ (since the distance from $x$ to $\partial G$ is less than $h^{1 / 2} \lambda$ ) and that if $x \in \partial G$, then $p=1$ (since in this case $x^{\pi}=x$ ).

Let a point $\left(t_{0}, x_{0}\right) \in Q$. We would like to find the value $u\left(t_{0}, x_{0}\right)$. Introduce a discretization of the interval $\left[t_{0}, T\right]$, for definiteness the equidistant one: $t_{0}<t_{1}<$ $\cdots<t_{N}=T, h:=\left(T-t_{0}\right) / N$.

In order to approximate the solution of system (1.4), we construct a Markov chain $\left(t_{m / 2}, X_{m / 2}\right)$ which stops when it reaches the boundary $\Gamma$ at a random step $\varkappa$. The number $m$ takes nonnegative integer values not greater than $2 N$ and then the index $m / 2$ takes integer and half-integer values. We put by definition that for odd $m=2 k+1$ the time $t_{m / 2}=t_{k+1 / 2}$ is equal to $t_{k}$, i.e., $X_{k}$ and $X_{k+1 / 2}$ belong to the same layer $t=t_{k}$. For brevity, we will often denote this chain as $X_{m / 2}$.

We take $X_{0}=x_{0}$. Let $\varkappa>k, k \leqq N-1$, and $X_{k} \in \bar{G}$ be known. We define $X_{k+1 / 2}$ as follows: If the state $X_{k} \notin S_{t_{k}, h}$, then $X_{k+1 / 2}=X_{k}$ (i.e., the point does not move), and if $X_{k} \in S_{t_{k}, h}$, then $X_{k+1 / 2}$ is the random variable taking two values: either $X_{k+1 / 2}=X_{k}^{\pi} \in \partial G$ with probability $p_{X_{k}, h}$ or $X_{k+1 / 2}=X_{k}+h^{1 / 2} \lambda n\left(X_{k}^{\pi}\right) \notin S_{t_{k}, h}$ with probability $q_{X_{k}, h}$. If $X_{k+1 / 2}=X_{k}^{\pi}$ (i.e., $\left(t_{k}, X_{k+1 / 2}\right) \in \Gamma$ ), then we put $\varkappa=k+\frac{1}{2}$, $X_{\varkappa}=X_{k}^{\pi}$, and the random walk is finished. If $\varkappa>k+\frac{1}{2}$, then $X_{k+1}$ is evaluated due to (2.1) for $t=t_{k}, x=X_{k+1 / 2}, \xi=\xi_{k}$ (we suppose that all $\xi_{k}$ are mutually independent and distributed as $\xi$ ). For $k=N-1$ and $\varkappa>N-\frac{1}{2}$ (i.e., on the last layer) $\varkappa=N$ since $\left(t_{N}, X_{N}\right)=\left(T, X_{N}\right) \in \Gamma$. Thus the chain $\left(t_{m / 2}, X_{m / 2}\right)$ has been constructed.

It is not difficult to see that $\left(t_{m / 2}, X_{m / 2}\right)$ remains in the domain $\bar{Q}$ with probability 1 , and $\varkappa$ either takes half-integer values from $\frac{1}{2}$ to $N-\frac{1}{2}$ or it is equal to $N$.

We also introduce an extended chain $\left(t_{m / 2}, X_{m / 2}, Y_{m / 2}, Z_{m / 2}\right)$. We put $Y_{0}=1$ and $Z_{0}=0$. Let $\varkappa \geqq k+\frac{1}{2}, k \leqq N-1$, and $Y_{k}, Z_{k}$ be known. Then, we put $Y_{k+1 / 2}=Y_{k}, Z_{k+1 / 2}=Z_{k}$. For $\varkappa>k+\frac{1}{2}, \quad Y_{k+1}$ and $Z_{k+1}$ are evaluated in accordance with system (2.1)-(2.3) for $t=t_{k}, x=X_{k+1 / 2}, y=Y_{k+1 / 2}=Y_{k}, z=$ $Z_{k+1 / 2}=Z_{k}, \xi=\xi_{k}$. Below we write the constructed algorithm formally.

Algorithm 1.

Step 0. $X_{0}=x_{0}, Y_{0}=1, Z_{0}=0, k=0$.

Step 1. $Y_{k+1 / 2}=Y_{k}, Z_{k+1 / 2}=Z_{k}$.

If $X_{k} \notin S_{t_{k}, h}$, then $X_{k+1 / 2}=X_{k}$ and go to Step 3 .

If $X_{k} \in S_{t_{k}, h}$, then either $X_{k+1 / 2}=X_{k}^{\pi}$ with probability $p_{X_{k}, h}$, or $X_{k+1 / 2}=$ $X_{k}+h^{1 / 2} \lambda n\left(X_{k}^{\pi}\right)$ with probability $q_{X_{k}, h}$.

Step 2. If $X_{k+1 / 2}=X_{k}^{\pi}$, then stop and $æ=k+\frac{1}{2}, X_{æ}=X_{k}^{\pi}, Y_{æ}=Y_{k+1 / 2}=Y_{k}$, $Z_{æ}=Z_{k+1 / 2}=Z_{k}$.

Step 3. Simulate $\xi_{k}$ and find $X_{k+1}, Y_{k+1}, Z_{k+1}$ according to (2.1)-(2.3) for $t=t_{k}$, $x=X_{k+1 / 2}, y=Y_{k+1 / 2}, z=Z_{k+1 / 2}, \xi=\xi_{k}$.

Step 4. If $k+1=N$, stop and $=N, X_{æ}=X_{N}, Y_{æ}=Y_{N}, Z_{æ}=Z_{N}$, otherwise $k=k+1$ and return to Step 1.

It may happen that it is more rational to choose both $h$ and $\lambda$ depending on chain's state: $h_{k}$ and $\lambda_{k}$. Then, in Theorem 1 (see below) one should put $h=\max _{0 \leqq k<N} h_{k}$. 
In practice, one can take $\lambda_{k}=\left|\sigma\left(t_{k}, X_{k}\right)\right|$, possibly with small corrections.

3. Convergence theorem. Denote by $\nu_{t_{0}, x_{0}}$ the number of those $t_{k}$ at which the chain gets into the set $S_{t_{k}, h}$. The event $\left\{\nu_{t_{0}, x_{0}}>n\right\}$ implies an event such that first $n$ trials in a certain trial scheme are unsuccessful ( $\Gamma$ is not attained). Moreover, the probability of each failure is less than $\frac{1}{2}$. Therefore, the following estimate takes place:

$$
\mathbf{P}\left\{\nu_{t_{0}, x_{0}}>n\right\} \leqq 2^{-n} .
$$

However, these arguments are not completely rigorous since the probability of exit of the considered chain $X_{m / 2}$ from $S_{t_{k}, h}$ depends on $X_{k}$. Along with the chain $X_{m / 2}$, let us consider a chain $X_{m / 2}^{\prime}$ which differs from the original one only in the following way: when $X_{m / 2}^{\prime}$ gets into the boundary set $S_{t_{k}, h}$, each time a coin is thrown so that the new chain hits the boundary at $X_{k}^{\pi}$ with probability $\frac{1}{2}$ and it hits the same state as the original chain with probability $\frac{1}{2}$, i.e., the state $X_{k}+h^{1 / 2} \lambda n\left(X_{k}^{\pi}\right) \notin S_{t_{k}, h}$. Since the new chain terminates with a smaller probability than the original chain, we have $\nu_{t_{0}, x_{0}} \leqq \nu_{t_{0}, x_{0}}^{\prime}$. Therefore, $\mathbf{P}\left\{\nu_{t_{0}, x_{0}}>n\right\} \leqq \mathbf{P}\left\{\nu_{t_{0}, x_{0}}^{\prime}>n\right\}$. However, the arguments before (3.1) are rigorous for $\nu_{t_{0}, x_{0}}^{\prime}$, i.e., $\mathbf{P}\left\{\nu_{t_{0}, x_{0}}^{\prime}>n\right\} \leqq 2^{-n}$. The last two inequalities imply (3.1). As a conclusion, we state the following lemma (in what follows we denote by the same letter $C$ various constants independent of, e.g., $t, x$, $y, z, m, h)$.

Lemma 1. Inequality (3.1) holds, together with the inequalities

$$
\begin{gathered}
\mathbf{P}\left\{\nu_{t_{0}, x_{0}}=n\right\} \leqq 2^{-(n-1)}, \\
\mathbf{E} \nu_{t_{0}, x_{0}} \leqq C, \quad \mathbf{E} \nu_{t_{0}, x_{0}}^{2} \leqq C,
\end{gathered}
$$

where $C$ does not depend on $t_{0}, x_{0}, h$.

We extend the definition of the constructed chain for all $m$ by the rule that if $m / 2>\varkappa$, then $\left(t_{m / 2}, X_{m / 2}, Y_{m / 2}, Z_{m / 2}\right)=\left(t_{\varkappa}, X_{\varkappa}, Y_{\varkappa}, Z_{\varkappa}\right)$. Now we prove a lemma on a one-step error. Introduce

$$
\begin{gathered}
d_{k}=u\left(t_{k+1 / 2}, X_{k+1 / 2}\right) Y_{k+1 / 2}+Z_{k+1 / 2}-u\left(t_{k}, X_{k}\right) Y_{k}-Z_{k}, \\
d_{k+1 / 2}=u\left(t_{k+1}, X_{k+1}\right) Y_{k+1}+Z_{k+1}-u\left(t_{k+1 / 2}, X_{k+1 / 2}\right) Y_{k+1 / 2}-Z_{k+1 / 2}, \\
k=0, \ldots, N-1 .
\end{gathered}
$$

Recall that $t_{k+1 / 2}=t_{k}, Y_{k+1 / 2}=Y_{k}, Z_{k+1 / 2}=Z_{k} ; X_{k+1 / 2}$ belongs to the layer $t=t_{k}$; the variable $d_{k}$ can be nonzero in the case of $X_{k} \in S_{t_{k}, h}$ only; if $\varkappa>k+\frac{1}{2}$, then $X_{k+1 / 2} \notin S_{t_{k}, h}$ and all the $2^{d}$ realizations of the random variable $X_{k+1}$ belong to $\bar{G}$; i.e., the standard (not boundary) step of the algorithm takes place. Clearly, if $\varkappa \leqq k+\frac{1}{2}$, then $d_{m / 2}=0, m \geqq 2 k+1$. We also note that it is not difficult to show that for sufficiently small $h$ the component $Y_{k}$ is positive.

Lemma 2. For an integer index $k$ the inequality

$$
\left|\mathbf{E}\left(d_{k} \mid X_{k}, Y_{k}, Z_{k}\right)\right| \leqq C h Y_{k} I_{S_{t_{k}}, h}\left(X_{k}\right) \chi_{æ \geqq k+1 / 2}
$$

holds. For a half-integer index $k+\frac{1}{2}$ the inequality

$$
\left|\mathbf{E}\left(d_{k+1 / 2} \mid X_{k+1 / 2}, Y_{k+1 / 2}, Z_{k+1 / 2}\right)\right| \leqq C h^{2} Y_{k} \chi_{æ>k+1 / 2}
$$

holds. 
Proof. Inequality (3.4) follows from the interpolation relation (2.4) and from the reminder before the lemma. Inequality (3.5) is a consequence of the fact that the one-step accuracy order of the Euler method (2.1)-(2.3) in the weak sense is $O\left(h^{2}\right)$.

THEOREM 1. Algorithm 1 has weak order of accuracy $O(h)$, i.e., the inequality

$$
\left|\mathbf{E}\left(\varphi\left(t_{æ}, X_{æ}\right) Y_{æ}+Z_{æ}\right)-u\left(t_{0}, x_{0}\right)\right| \leqq C h
$$

holds with $C$ independent of $t_{0}, x_{0}$, and $h$.

Proof. We have

$$
\begin{aligned}
R & :=\mathbf{E}\left(\varphi\left(t_{æ}, X_{æ}\right) Y_{æ}+Z_{æ}\right)-u\left(t_{0}, x_{0}\right)=\mathbf{E}\left(u\left(t_{æ}, X_{æ}\right) Y_{æ}+Z_{æ}\right)-u\left(t_{0}, x_{0}\right) \\
= & \mathbf{E} \sum_{m=0}^{2 æ-1}\left(u\left(t_{(m+1) / 2}, X_{(m+1) / 2}\right) Y_{(m+1) / 2}+Z_{(m+1) / 2}\right. \\
& \left.\quad-u\left(t_{m / 2}, X_{m / 2}\right) Y_{m / 2}-Z_{m / 2}\right) \\
= & \sum_{k=0}^{2 N-1} \mathbf{E} d_{m / 2}=\sum_{k=0}^{N-1} \mathbf{E} d_{k+1 / 2}+\sum_{k=0}^{N-1} \mathbf{E} d_{k} \\
= & \sum_{k=0}^{N-1} \mathbf{E E}\left(d_{k+1 / 2} \mid X_{k+1 / 2}, Y_{k+1 / 2}, Z_{k+1 / 2}\right)+\sum_{k=0}^{N-1} \mathbf{E E}\left(d_{k} \mid X_{k}, Y_{k}, Z_{k}\right) .
\end{aligned}
$$

Due to (3.5), the absolute value of the first sum is estimated by $C h^{2} \sum_{k=0}^{N-1} \mathbf{E} Y_{k}$. It is not difficult to show that $\mathbf{E} Y_{k} \leqq C, k=0, \ldots, N-1$. Thus, the first sum is $O(h)$ uniformly in $t_{0}, x_{0}, h$. Using (3.4) and Lemma 1, we estimate the second sum:

$$
\begin{aligned}
& \left|\sum_{k=0}^{N-1} \mathbf{E E}\left(d_{k} \mid X_{k}, Y_{k}, Z_{k}\right)\right| \leqq C h \mathbf{E} \sum_{k=0}^{N-1}\left(Y_{k} I_{S_{t_{k}, h}}\left(X_{k}\right) \chi_{æ \geqq k+1 / 2}\right) \\
& \quad \leqq C h \mathbf{E}\left(\max _{0 \leqq k \leqq N-1} Y_{k} \cdot \sum_{k=0}^{N-1} I_{S_{t_{k}, h}}\left(X_{k}\right)\right)=C h \mathbf{E}\left(\max _{0 \leqq k \leqq N-1} Y_{k} \cdot \nu_{t_{0}, x_{0}}\right) \\
& \quad \leqq C h\left(\mathbf{E} \max _{0 \leqq k \leqq N-1} Y_{k}^{2}\right)^{1 / 2}\left(\mathbf{E} \nu_{t_{0}, x_{0}}^{2}\right)^{1 / 2} \leqq C h\left(\mathbf{E} \max _{0 \leqq k \leqq N-1} Y_{k}^{2}\right)^{1 / 2} .
\end{aligned}
$$

Let $c(t, x) \leqq \bar{c},(t, x) \in \bar{Q}$, where $\bar{c}$ is a constant. Introduce the sequence

$$
\begin{array}{ll}
Y_{0}^{\prime} & =1, \quad Y_{k}^{\prime}=Y_{k-1}^{\prime}\left(1+h \bar{c}+h^{1 / 2} \mu\left(t_{k-1}, X_{k-1 / 2}\right) \xi_{k-1}\right), \quad k \leqq æ ; \\
Y_{k}^{\prime} & =Y_{æ}^{\prime}, \quad k>æ .
\end{array}
$$

It is obvious that $Y_{k} \leqq Y_{k}^{\prime}$. It is not difficult to see that the sequence $V_{k}=(1+h \bar{c})^{-k} Y_{k}^{\prime}$ is a martingale. Hence

$$
\mathbf{E} \max _{1 \leqq k \leqq N-1} V_{k}^{2} \leqq 4 \mathbf{E} V_{N-1}^{2} \leqq C
$$

(as before, here $C$ is independent of $h$ ), and this together with (3.7) implies (3.6). Theorem 1 is proved.

We note that despite some steps of Algorithm 1 being very rough (their onestep errors are $O(h))$, this algorithm converges, and, moreover, its global order of convergence is $O(h)$. We have this rate of convergence due to the fact that the number of rough steps (on average) is bounded from above by a constant which does not grow as $h \rightarrow 0$. 


\section{Other simplest random walks.}

4.1. Algorithm 2. The next algorithm is obtained by a simplification of Algorithm 1. Indeed, as soon as $X_{k}$ gets into the boundary domain $S_{t_{k}, h}$, the random walk terminates, i.e., $\varkappa=k$, and $\bar{X}_{\varkappa}=X_{k}^{\pi}, Y_{\varkappa}=Y_{k}, Z_{\varkappa}=Z_{k}$ is taken as the final state of the Markov chain. In this case we do not need values of the chain with half-integer indices. Let us write this algorithm formally.

Algorithm 2.

Step 0. $X_{0}=x_{0}, Y_{0}=1, Z_{0}=0, k=0$.

Step 1. If $X_{k} \notin S_{t_{k}, h}$, then go to Step 2.

If $X_{k} \in S_{t_{k}, h}$, then stop and $=k, \bar{X}_{æ}=X_{k}^{\pi}, Y_{æ}=Y_{k}, Z_{æ}=Z_{k}$.

Step 2. Simulate $\xi_{k}$ and find $X_{k+1}, Y_{k+1}, Z_{k+1}$ according to (2.1)-(2.3) for $t=t_{k}$, $x=X_{k}, y=Y_{k}, z=Z_{k}, \xi=\xi_{k}$.

Step 3. If $k+1=N$, stop and $=N, \bar{X}_{æ}=X_{N}, Y_{æ}=Y_{N}, Z_{æ}=Z_{N}$, otherwise $k=k+1$ and return to Step 1 .

In this algorithm, one-step errors of all steps except the last one are $O\left(h^{2}\right)$. The error on the last step (i.e., on the step $\varkappa$ ) is estimated as $O(\sqrt{h})$. It is easy to prove the following theorem (we note in passing that the conditions on smoothness of the solution and parameters mentioned in the introduction can be weakened for this theorem).

TheOREM 2. Algorithm 2 has weak order of accuracy $O(\sqrt{h})$,

$$
\left|\mathbf{E}\left(\varphi\left(t_{æ}, \bar{X}_{æ}\right) Y_{æ}+Z_{æ}\right)-u\left(t_{0}, x_{0}\right)\right| \leqq C \sqrt{h},
$$

where $C$ is independent of $t_{0}, x_{0}$, and $h$.

A similar random walk was proposed in [7]. In contrast to Algorithm 2, which terminates when the chain enters the boundary domain $S_{t_{k}, h} \subset \bar{G}$, the method from [7] terminates when the chain exits from $\bar{G}$ and the projection of the point, having exited from the domain, on the boundary $\partial G$ is taken as the final state of the chain.

4.2. Algorithm 3. Now we construct a random walk with a more accurate onestep approximation for $x \in S_{t, h}$ than in Algorithm 1. Let $x \in S_{t, h}$. We denote by $\xi^{(\alpha)}=\left(\xi^{(\alpha), 1}, \ldots, \xi^{(\alpha), d}\right)^{\top}, \alpha=1, \ldots, 2^{d}$, the values of the vector $\xi$ from (2.1), and we assign indices to these values so that $\xi^{\left(2^{d-1}+\alpha\right)}=-\xi^{(\alpha)}, \alpha=1, \ldots, 2^{d-1}$. We denote by $X^{(\alpha)}$ the value of the vector $X$ from (2.1) corresponding to $\xi^{(\alpha)}$. At least one of the points $X^{(\alpha)} \notin \bar{G}$ since $x \in S_{t, h}$. We connect the point $x$ with those $X^{(\alpha)}$ which are outside $\bar{G}$ by the curves $\eta^{(\alpha)}(\theta)$ :

$$
\eta^{(\alpha)}(\theta)=\left(x+\theta h(b(t, x)-\sigma(t, x) \mu(t, x))+\sqrt{\theta h} \sigma(t, x) \xi^{(\alpha)}\right), \quad \theta \in[0,1] .
$$

There is a value $\theta=\theta^{(\alpha)}, 0<\theta^{(\alpha)}<1$, (it is unique since $h$ is sufficiently small and $\partial G$ is smooth) such that the point $\eta^{(\alpha)}:=\eta^{(\alpha)}\left(\theta^{(\alpha)}\right)$ belongs to the boundary $\partial G$. We put $\theta^{(\alpha)}=1$ and $\eta^{(\alpha)}=X^{(\alpha)}$ for those points $X^{(\alpha)}$ which belong to $\bar{G}$.

Introduce the pair

$$
\left(\vartheta^{(\alpha)}, \eta^{(\alpha)}\right):=\left(t+\theta^{(\alpha)} h, \eta^{(\alpha)}\right) .
$$

Here $\alpha$ is the random variable taking values $\left\{1, \ldots, 2^{d}\right\}$ and distributed by the law

$$
\begin{aligned}
& p_{i}=\mathbf{P}\{\alpha=i\}=\frac{\gamma}{\sqrt{\theta^{(i)}}\left(\sqrt{\theta^{(i)}}+\sqrt{\theta^{\left(2^{d-1}+i\right)}}\right)}, \quad i=1, \ldots, 2^{d-1}, \\
& p_{i}=\mathbf{P}\{\alpha=i\}=\frac{\gamma}{\sqrt{\theta^{(i)}}\left(\sqrt{\theta^{(i)}}+\sqrt{\left.\theta^{\left(i-2^{d-1}\right.}\right)},\right.}, \quad i=2^{d-1}+1, \ldots, 2^{d},
\end{aligned}
$$


where $\gamma$ is uniquely found from the condition $\sum_{i=1}^{2^{d}} \mathbf{P}\{\alpha=i\}=1$.

Now we construct a Markov chain $\left(\vartheta_{k}, X_{k}\right)$ which stops reaching the boundary $\Gamma$ at a random step $\varkappa$. The index $k$ takes nonnegative integer values not greater than $N$, and $\vartheta_{k}=t_{k}$, at least for $k<\varkappa$. We put $\vartheta_{0}=t_{0}, X_{0}=x_{0}$. Let $\left(\vartheta_{k}, X_{k}\right) \in Q$, i.e., $\varkappa>k, k \leqq N-1, \vartheta_{k}=t_{k}$. Let us define $\left(\vartheta_{k+1}, X_{k+1}\right)$. If $X_{k} \notin S_{t_{k}, h}$, then $\vartheta_{k+1}=t_{k+1}$ and $X_{k+1}$ is found in accordance with (2.1) for $t=t_{k}, x=X_{k}, \xi=\xi_{k}$. If $X_{k} \in S_{t_{k}, h}$, then

$$
\left(\vartheta_{k+1}, X_{k+1}\right)=\left(\vartheta_{k}^{\left(\alpha_{k}\right)}, \eta_{k}^{\left(\alpha_{k}\right)}\right),
$$

where $\left(\vartheta_{k}^{\left(\alpha_{k}\right)}, \eta_{k}^{\left(\alpha_{k}\right)}\right)$ is found as $\left(\vartheta^{(\alpha)}, \eta^{(\alpha)}\right)$ from (4.1) for $t=t_{k}, x=X_{k}, \alpha=\alpha_{k}$ (both $\xi_{k}$ and $\alpha_{k}$ are independent of previous history; it is also clear that at each step we simulate either $\xi_{k}$ or $\alpha_{k}$ ). In this case, due to the construction of the pair $\left(\vartheta_{k}^{\left(\alpha_{k}\right)}, X_{k}^{\left(\alpha_{k}\right)}\right)$, the point $X_{k+1}$ either belongs to $\partial G$ with probability $p \geqq 2^{-d}$ and the random walk terminates (in this case $\varkappa=k+1,\left(\vartheta_{\varkappa}, X_{\varkappa}\right)=\left(\vartheta_{k+1}, X_{k+1}\right)$ ) or $X_{k+1} \notin \partial G$ (this means that the realized $\alpha_{k}$ is such that $\theta_{k}^{\left(\alpha_{k}\right)}=1$ and, therefore, $\left.\vartheta_{k+1}=t_{k+1}\right)$ and for $k+1<N$ the random walk should be continued. For $k+1=N$ the point $\left(\vartheta_{k+1}, X_{k+1}\right) \in \Gamma$ and the random walk terminates with $\varkappa=N,\left(\vartheta_{\varkappa}, X_{\varkappa}\right)=$ $\left(T, X_{N}\right)$. Thus, the chain $\left(\vartheta_{k}, X_{k}\right)$ has been constructed.

Note that $\left(\vartheta_{k}, X_{k}\right)$ remains in the domain $\bar{Q}$ with probability $1 ; \varkappa$ takes nonnegative integer values not greater than $N$.

Now we introduce an extended Markov chain $\left(\vartheta_{k}, X_{k}, Y_{k}, Z_{k}\right)$. We put $Y_{0}=1$, $Z_{0}=0$. Let $\varkappa>k$ and $Y_{k}, Z_{k}$ be known. If $X_{k} \notin S_{t_{k}, h}$, then $Y_{k+1}, Z_{k+1}$ are evaluated in accordance with (2.1)-(2.3) for $t=t_{k}, x=X_{k}, y=Y_{k}, z=Z_{k}, \xi=\xi_{k}$. If $X_{k} \in S_{t_{k}, h}$, then

$$
\begin{aligned}
& Y_{k+1}=Y_{k}+\theta_{k}^{\left(\alpha_{k}\right)} h c\left(\vartheta_{k}, X_{k}\right) Y_{k}+\sqrt{\theta_{k}^{\left(\alpha_{k}\right)} h} \mu^{\mathrm{T}}\left(\vartheta_{k}, X_{k}\right) Y_{k} \xi^{\left(\alpha_{k}\right)}, \\
& Z_{k+1}=Z_{k}+\theta_{k}^{\left(\alpha_{k}\right)} h g\left(\vartheta_{k}, X_{k}\right) Y_{k}+\sqrt{\theta_{k}^{\left(\alpha_{k}\right)} h} F^{\mathrm{T}}\left(\vartheta_{k}, X_{k}\right) Y_{k} \xi^{\left(\alpha_{k}\right)},
\end{aligned}
$$

where $\theta_{k}^{(\alpha)}, \alpha=1, \ldots, 2^{d}$, and $\alpha_{k}$ are the same as in the evaluation of $\left(\vartheta_{k+1}, X_{k+1}\right)$.

Not writing the constructed algorithm formally, we shall call it Algorithm 3. We prove the following lemma on a one-step error of Algorithm 3.

LEMma 3. The following inequality is valid:

$$
\begin{aligned}
& \left|\mathbf{E}\left(u\left(\vartheta_{k+1}, X_{k+1}\right) Y_{k+1}+Z_{k+1}-u\left(\vartheta_{k}, X_{k}\right) Y_{k}-Z_{k} \mid X_{k}, Y_{k}, Z_{k}\right)\right| \\
& \quad \leqq C h^{2} Y_{k} I_{G \backslash S_{t_{k}, h}}\left(X_{k}\right) \chi_{æ>k}+C h^{3 / 2} Y_{k} I_{S_{t_{k}, h}}\left(X_{k}\right) \chi_{æ>k} .
\end{aligned}
$$

Proof. For $X_{k} \in G \backslash S_{t_{k}, h}$, inequality (4.3) is a consequence of the fact that the one-step accuracy order of the Euler method (2.1)-(2.3) in the weak sense is $O\left(h^{2}\right)$.

Let $X_{k} \in S_{t_{k}, h}$. Then we have

$$
\begin{aligned}
\rho & :=\mathbf{E}\left(u\left(\vartheta_{k+1}, X_{k+1}\right) Y_{k+1}+Z_{k+1}-u\left(\vartheta_{k}, X_{k}\right) Y_{k}-Z_{k} \mid X_{k}, Y_{k}, Z_{k}\right) \\
& =\sum_{\alpha=1}^{2^{d}} p_{\alpha}\left(u\left(\vartheta_{k+1}^{(\alpha)}, X_{k+1}^{(\alpha)}\right) Y_{k+1}^{(\alpha)}+Z_{k+1}^{(\alpha)}\right)-u\left(\vartheta_{k}, X_{k}\right) Y_{k}-Z_{k},
\end{aligned}
$$

where $p_{\alpha}$ is from (4.2). 
Substituting the expressions for $\vartheta_{k+1}^{(\alpha)}, X_{k+1}^{(\alpha)}, Y_{k+1}^{(\alpha)}, Z_{k+1}^{(\alpha)}$ in (4.4) and expanding $u\left(\vartheta_{k+1}^{(\alpha)}, X_{k+1}^{(\alpha)}\right)$ in a series in powers of $h$ around the point $\left(\vartheta_{k}, X_{k}\right)$, we obtain

$$
\begin{aligned}
& \rho=\sum_{\alpha=1}^{2^{d}} p_{\alpha} Y_{k}\{ {\left[u\left(\vartheta_{k}, X_{k}\right)+\frac{\partial u}{\partial t} \theta_{k}^{(\alpha)} h\right.} \\
&+\sum_{j=1}^{d} \frac{\partial u}{\partial x^{j}}\left(\theta_{k}^{(\alpha)} h\left(b^{j}-(\sigma \mu)^{j}\right)+\sqrt{\theta_{k}^{(\alpha)} h}\left(\sigma \xi^{(\alpha)}\right)^{j}\right) \\
&\left.+\frac{1}{2} \sum_{j, l=1}^{d} \frac{\partial^{2} u}{\partial x^{j} \partial x^{l}} \theta_{k}^{(\alpha)} h\left(\sigma \xi^{(\alpha)}\right)^{j}\left(\sigma \xi^{(\alpha)}\right)^{l}+O\left(h^{3 / 2}\right)\right] \\
&\left.\times\left(1+\theta_{k}^{(\alpha)} h c+\sqrt{\theta_{k}^{(\alpha)} h} \mu^{\mathrm{T}} \xi^{(\alpha)}\right)+\left(\theta_{k}^{(\alpha)} h g+\sqrt{\theta_{k}^{(\alpha)} h} F^{\mathrm{T}} \xi^{(\alpha)}\right)\right\} \\
&+\sum_{\alpha=1}^{2^{d}} p_{\alpha} Z_{k}-u\left(\vartheta_{k}, X_{k}\right) Y_{k}-Z_{k},
\end{aligned}
$$

where the derivatives of $u$ and the coefficients $b, \sigma, \mu, c, g, F$ are evaluated at the point $\left(\vartheta_{k}, X_{k}\right)$.

Rearranging the terms in (4.5), we get

$$
\begin{aligned}
\rho= & Y_{k} h \sum_{\alpha=1}^{2^{d}} p_{\alpha} \theta_{k}^{(\alpha)}\left[\frac{\partial u}{\partial t}+\frac{1}{2} \sum_{j, l=1}^{d} \frac{\partial^{2} u}{\partial x^{j} \partial x^{l}} \sum_{m=1}^{d} \sigma_{j m} \sigma_{l m}+\sum_{j=1}^{d} \frac{\partial u}{\partial x^{j}} b^{j}+c u+g\right] \\
& +Y_{k} \sqrt{h} \sum_{\alpha=1}^{2 d} p_{\alpha} \sqrt{\theta_{k}^{(\alpha)}}\left[\sum_{j=1}^{d} \frac{\partial u}{\partial x^{j}}\left(\sigma \xi^{(\alpha)}\right)^{j}+u \mu^{\mathrm{T}} \xi^{(\alpha)}+F^{\mathrm{T}} \xi^{(\alpha)}\right] \\
& -Y_{k} h \sum_{j=1}^{d} \frac{\partial u}{\partial x^{j}}(\sigma \mu)^{j} \sum_{\alpha=1}^{2^{d}} p_{\alpha} \theta_{k}^{(\alpha)}+Y_{k} h \sum_{j=1}^{d} \frac{\partial u}{\partial x^{j}} \sum_{\alpha=1}^{2^{d}} p_{\alpha} \theta_{k}^{(\alpha)} \mu^{\mathrm{T}} \xi^{(\alpha)}\left(\sigma \xi^{(\alpha)}\right)^{j} \\
& +\frac{1}{2} Y_{k} h \sum_{j, l=1}^{d} \frac{\partial^{2} u}{\partial x^{j} \partial x^{l}} \sum_{n, m=1, n \neq m}^{d} \sum_{\alpha=1}^{2^{d}} p_{\alpha} \theta_{k}^{(\alpha)} \sigma_{j n} \xi^{(\alpha), n} \sigma_{l m} \xi^{(\alpha), m}+O\left(h^{3 / 2}\right) .
\end{aligned}
$$

The first sum in (4.6) is equal to zero since $u(t, x)$ satisfies (1.1). We have from (4.2) that $p_{\alpha} \sqrt{\theta_{k}^{(\alpha)}}=p_{2^{d-1}+\alpha} \sqrt{\theta_{k}^{\left(2^{d-1}+\alpha\right)}}, \alpha=1, \ldots, 2^{d-1}$, and, therefore, for any $r=1, \ldots, d$ we obtain

$$
\sum_{\alpha=1}^{2^{d}} p_{\alpha} \sqrt{\theta_{k}^{(\alpha)}} \xi^{(\alpha), r}=\sum_{\alpha=1}^{2^{d-1}} \xi^{(\alpha), r}\left(p_{\alpha} \sqrt{\theta_{k}^{(\alpha)}}-p_{\left(2^{d-1}+\alpha\right)} \sqrt{\theta_{k}^{\left(2^{d-1}+\alpha\right)}}\right)=0
$$

whence the second sum in (4.6) is equal to zero. It follows from (4.2) that $p_{\alpha} \theta_{k}^{(\alpha)}+$ $p_{2^{d-1}+\alpha} \theta_{k}^{\left(2^{d-1}+\alpha\right)}=\gamma$. Then it is not difficult to check that for $n \neq m$

$$
\sum_{\alpha=1}^{2^{d}} p_{\alpha} \theta_{k}^{(\alpha)} \xi^{(\alpha), n} \xi^{(\alpha), m}=\gamma \sum_{\alpha=1}^{2^{d-1}} \xi^{(\alpha), n} \xi^{(\alpha), m}=\frac{\gamma}{2} \sum_{\alpha=1}^{2^{d}} \xi^{(\alpha), n} \xi^{(\alpha), m}=0
$$


This implies that the last sum in (4.6) is equal to zero and the sum before the last one is equal to $Y_{k} h \sum_{j=1}^{d} \frac{\partial u}{\partial x^{j}}(\sigma \mu)^{j} \sum_{\alpha=1}^{2^{d}} p_{\alpha} \theta_{k}^{(\alpha)}$. Thus, $\rho=O\left(h^{3 / 2}\right)$. Lemma 3 is proved.

The proof of the next theorem is similar to the proof of Theorem 1.

TheOREM 3. Algorithm 3 has weak order of accuracy $O(h)$,

$$
\left|\mathbf{E}\left(\varphi\left(\vartheta_{æ}, X_{æ}\right) Y_{æ}+Z_{æ}\right)-u\left(t_{0}, x_{0}\right)\right| \leqq C h,
$$

where $C$ is independent of $t_{0}, x_{0}, h$.

We note that the chain $\left(\vartheta_{k}, X_{k}, Y_{k}, Z_{k}\right)$ is more expensive from a computational point of view, but due to smaller errors in the boundary domain, it allows us to obtain more accurate results than the chain $\left(t_{m / 2}, X_{m / 2}, Y_{m / 2}, Z_{m / 2}\right)$ from section 2 .

Remark 1. The one-step approximation of Algorithm 3 is used in [8] to construct a layer (deterministic) method for solving the Dirichlet problem for semilinear parabolic equations.

5. The Dirichlet problem for an elliptic equation. Consider the Dirichlet problem for an elliptic equation

$$
\begin{gathered}
\frac{1}{2} \sum_{i, j=1}^{d} a^{i j}(x) \frac{\partial^{2} u}{\partial x^{i} \partial x^{j}}+\sum_{i=1}^{d} b^{i}(x) \frac{\partial u}{\partial x^{i}}+c(x) u+g(x)=0, \quad x \in G, \\
\left.u\right|_{\partial G}=\varphi(x) .
\end{gathered}
$$

We assume that the coefficients $a^{i j}=a^{j i}$ satisfy the strict ellipticity condition in $\bar{G}$ and also that conditions hold which guarantee existence of the unique solution $u(x)$ of problem $(5.1)-(5.2)$ from the class $C^{4}(\bar{G})$. We recall [9] that it is sufficient for the above to require that the functions $a^{i j}(x), b^{i}(x), c(x), g(x)$ are from the class $C^{2}(\bar{G}), G$ is an open domain with a twice continuously differentiable boundary $\partial G, \varphi(x) \in C^{4}(\bar{G})$, and $c(x) \leqq 0, x \in \bar{G}$.

The solution of problem (5.1)-(5.2) has the probabilistic representation:

$$
u(x)=\mathbf{E}\left[\varphi\left(X_{x}(\tau)\right) Y_{x, 1}(\tau)+Z_{x, 1,0}(\tau)\right],
$$

where $X_{x}(s), Y_{x, y}(s), Z_{x, y, z}(s), s \geqq 0$, is the solution of the Cauchy problem for the system of SDEs:

$$
\begin{aligned}
d X & =b(X) d s+\sigma(X) d w(s), & X(0) & =x, \\
d Y & =c(X) Y d s, & Y(0) & =y, \\
d Z & =g(X) Y d s+F^{\mathrm{T}}(X) Y d w(s), & Z(0) & =z,
\end{aligned}
$$

$x \in G$, and $\tau=\tau_{x}$ is the first exit time of the trajectory $X_{x}(s)$ to the boundary $\partial G$.

The notation here and in what follows is similar to the notation in sections 1 and 2 . In this section we restrict ourselves to the case $\mu \equiv 0$.

First we construct an algorithm of random walk for problem (5.1)-(5.2), which is similar to Algorithm 1. We apply the weak explicit Euler approximation with the simplest simulation of noise to system (5.4)-(5.6):

$$
\begin{aligned}
X_{t, x}(t+h) & \approx X=x+h b(x)+h^{1 / 2} \sigma(x) \xi, \\
Y_{t, x, y}(t+h) & \approx Y=y+h c(x) y, \\
Z_{t, x, y, z}(t+h) & \approx Z=z+h g(x) y+h^{1 / 2} F^{\mathrm{T}}(x) y \xi .
\end{aligned}
$$


Introduce the set of boundary points $S_{h} \subset \bar{G}: x \in S_{h}$ if at least one of the $2^{d}$ values of the vector $X$ is outside $\bar{G}$. Let a constant $\lambda>0$ be such that if the distance from $x$ to the boundary $\partial G$ is equal to or greater than $\lambda \sqrt{h}$, then $x$ is not a boundary point and, therefore, for such $x$ all the realizations of the random variable $X$ belong to $\bar{G}$.

Let $x \in S_{h}$. Introduce the random vector $X_{x, h}^{\pi}$ taking two values $x^{\pi}$ and $x+$ $h^{1 / 2} \lambda n\left(x^{\pi}\right)$ with probabilities $p=p_{x, h}$ and $q=q_{x, h}=1-p_{x, h}$, respectively, where

$$
p_{x, h}=h^{1 / 2} \frac{\lambda}{\left|x+h^{1 / 2} \lambda n\left(x^{\pi}\right)-x^{\pi}\right|},
$$

$x^{\pi} \in \partial G$ is the projection of the point $x$ on the boundary $\partial G$, and $n\left(x^{\pi}\right)$ is the unit vector of internal normal to $\partial G$ at $x^{\pi}$.

To approximate the solution of system (5.4), we construct a Markov chain $X_{m / 2}$ which stops when it reaches the boundary $\partial G$ at a random step $\varkappa$. The number $m$ takes nonnegative integer values and then the index $m / 2$ takes integer and half-integer values.

We take $X_{0}=x_{0}$. Let $\varkappa>k$ and $X_{k} \in G$ be known. We define $X_{k+1 / 2}$ as follows. If the state $X_{k} \notin S_{h}$, then $X_{k+1 / 2}=X_{k}$ (the point does not move) and we continue the random walk, i.e., $\varkappa>k+\frac{1}{2}$. If $X_{k} \in S_{h}$, then we put $X_{k+1 / 2}=X_{X_{k}, h}^{\pi}$. In this case $X_{k+1 / 2}$ can take two values: either $X_{k}^{\pi} \in \partial G$ with probability $p_{X_{k}, h}$ or $X_{k}+h^{1 / 2} \lambda n\left(X_{k}^{\pi}\right) \notin S_{h}$ with probability $q_{X_{k}, h}$. If $X_{k+1 / 2}=X_{k}^{\pi}$ (i.e., $X_{k+1 / 2} \in \partial G$ ), then we put $\varkappa=k+\frac{1}{2}, X_{\varkappa}=X_{k}^{\pi}$, and the random walk terminates. If $X_{k+1 / 2}=$ $X_{k}+h^{1 / 2} \lambda n\left(X_{k}^{\pi}\right)$, then the random walk is continued, i.e., $\varkappa>k+\frac{1}{2}$. For $\varkappa>k+\frac{1}{2}$ the value of $X_{k+1}$ is found according to (5.7) with $x=X_{k+1 / 2}, \xi=\xi_{k}$. Thus, the chain $X_{m / 2}$ has been constructed.

It is not difficult to see that $X_{m / 2}$ remains in the domain $\bar{G}$ with probability 1 and $\varkappa$ takes half-integer values.

We also introduce an extended chain $\left(X_{m / 2}, Y_{m / 2}, Z_{m / 2}\right)$. We put $Y_{0}=1$ and $Z_{0}=0$. Let $\varkappa \geqq k+\frac{1}{2}$ and $Y_{k}, Z_{k}$ be known. Then, we put $Y_{k+1 / 2}=Y_{k}$, $Z_{k+1 / 2}=Z_{k}$. For $\varkappa>k+\frac{1}{2}$, the values $Y_{k+1}, Z_{k+1}$ are evaluated in accordance with system (5.7)-(5.9) for $x=X_{k+1 / 2}, y=Y_{k+1 / 2}=Y_{k}, z=Z_{k+1 / 2}=Z_{k}, \xi=\xi_{k}$.

We shall call the constructed algorithm Algorithm $1^{\prime}$ since it is similar to Algorithm 1 for a parabolic equation. We emphasize that here $\varkappa$ can take arbitrary large values in contrast to Algorithm 1.

Denote by $\nu_{x_{0}}$ the number of those $k$ at which the chain enters the set $S_{h}$.

LEMma 4. The following inequalities hold:

$$
\begin{gathered}
\mathbf{P}\left\{\nu_{x_{0}}=n\right\} \leqq 2^{-(n-1)}, \quad \mathbf{E} \nu_{x_{0}} \leqq C, \\
\mathbf{E} \rightsquigarrow \frac{C}{h},
\end{gathered}
$$

where $C$ does not depend on $t_{0}$ and $h$.

Proof. Inequalities (5.10) are proved analogously to Lemma 1. We will prove inequalities (5.11) using the technique of estimating mean number of steps from [10] and [11]. Denote by $\mu_{x}$ the number of steps which the chain $X_{m / 2}$ starting from $x \in G \backslash S_{h}$ spends in the domain $G \backslash S_{h}$ before it gets into $S_{h}$. In the domain $G \backslash S_{h}$ we have $X_{k+1 / 2}=X_{k}$. Therefore, the number of steps, which $X_{k}$ spends in the domain $G \backslash S_{h}$, coincides with $\mu_{x} / 2$. Note that $X_{k}$ is a chain up to the moment of entering $S_{h}$ inclusively. In connection with the chain $X_{k}$ we consider the boundary 
value problem

$$
\begin{array}{ll}
P V-V=-f(x), & x \in G \backslash S_{h}, \\
V(x)=0, & x \in S_{h},
\end{array}
$$

where $P$ is the one-step transition operator: $P V(x)=\mathbf{E} V\left(X_{1}\right), X_{0}=x$.

It is known [12, p. 322] that the solution of this problem is the function

$$
V(x)=\mathbf{E} \sum_{k=0}^{\mu_{x}-1} f\left(X_{k}\right)
$$

If we take $f(x)$ equal to the indicator function $f(x)=I_{G \backslash S_{h}}(x)$, then the solution of problem (5.12)-(5.13) is $V(x)=\mathbf{E} \mu_{x}$. Further, if we find the solution $V(x)$ of (5.12)-(5.13) with a function $f(x)$ which everywhere in $G \backslash S_{h}$ satisfies the condition

$$
f(x) \geqq I_{G \backslash S_{h}}(x),
$$

then, due to (5.14),

$$
\mathbf{E} \mu_{x} \leqq V(x) .
$$

We take $V(x)$ of the form (see [13, p. 132])

$$
V(x)= \begin{cases}A^{2}-|x+B|^{2 n}, & x \in G \backslash S_{h}, \\ 0, & x \in S_{h},\end{cases}
$$

where $B$ is a $d$-dimensional vector such that

$$
\min _{x \in \bar{G}}|x+B| \geqq C>0,
$$

$n$ is a sufficiently large natural number (how to choose it is shown below), and $A^{2}=$ $\max _{x \in \bar{G}}|x+B|^{2 n}$. The function $V(x)$ satisfies the boundary condition (5.13).

Let $x \in G \backslash S_{h}$; then $X_{1}=x+h b(x)+h^{1 / 2} \sigma(x) \xi$. It is not difficult to obtain (recall that $a=\sigma \sigma^{\top}$ )

$$
\begin{aligned}
P V(x)-V(x)=- & h n|x+B|^{2 n-4} \\
\times & {\left[2|x+B|^{2}(x+B, b(x))+|x+B|^{2} \sum_{i=1}^{d} a^{i i}\right.} \\
& \left.\quad+2(n-1) \sum_{i, j=1}^{d} a^{i j}(x)\left(x^{i}+B^{i}\right)\left(x^{j}+B^{j}\right)\right]+O\left(h^{2}\right) .
\end{aligned}
$$

Since the coefficients $a^{i j}=a^{j i}$ of (5.1) satisfy the strict ellipticity condition in $\bar{G}$, we have, for all $x \in \bar{G}$ and an arbitrary $d$-dimensional vector $y$,

$$
\sum_{i, j=1}^{d} a^{i j}(x) y^{i} y^{j} \geqq \lambda_{1}^{2} \sum_{i=1}^{d}\left(y^{i}\right)^{2}, \quad \lambda_{1}^{2}=\min _{x \in \bar{G}} \min _{1 \leqq i \leqq d} \lambda_{i}^{2}(x)>0,
$$

where $\lambda_{1}^{2}(x) \leqq \cdots \leqq \lambda_{d}^{2}(x)$ are eigenvalues of the matrix $a(x)=\left\{a^{i j}(x)\right\}$. 
Relations (5.19) and (5.20) imply

$$
P V-V \leqq-h n|x+B|^{2 n-2}\left[2(x+B, b(x))+(2 n-2+d) \lambda_{1}^{2}\right]+O\left(h^{2}\right) .
$$

We select $n$ so that for all $x \in \bar{G}$,

$$
2(x+B, b(x))+(2 n-2+d) \lambda_{1}^{2} \geqq C>0
$$

is always possible. Then, for sufficiently small $h$ we obtain $P V-V \leqq-\gamma h$, where $\gamma>0$ is independent of $h$ and $x$.

Obviously, the function $v(x)=V(x) /(\gamma h)$ is the solution of problem (5.12)-(5.13) with $f(x) \geqq 1$. Therefore, (see (5.15)-(5.17)) $\mathbf{E} \mu_{x} \leqq A^{2} /(\gamma h)$. From here and inequalities (5.10), it is not difficult to obtain the estimate (5.11). Lemma 4 is proved.

We extend the definition of the constructed chain for all $m$ by the following rule: If $m / 2>\varkappa$, then $\left(X_{m / 2}, Y_{m / 2}, Z_{m / 2}\right)=\left(X_{\varkappa}, Y_{\varkappa}, Z_{\varkappa}\right)$. Introduce

$$
\begin{aligned}
d_{k} & =u\left(X_{k+1 / 2}\right) Y_{k+1 / 2}+Z_{k+1 / 2}-u\left(t_{k}, X_{k}\right) Y_{k}-Z_{k}, \\
d_{k+1 / 2} & =u\left(X_{k+1}\right) Y_{k+1}+Z_{k+1}-u\left(X_{k+1 / 2}\right) Y_{k+1 / 2}-Z_{k+1 / 2}, \quad k=0,1, \ldots .
\end{aligned}
$$

The lemma on one-step errors is proved analogously to Lemma 2.

Lemma 5. For an integer index $k$, the inequality

$$
\left|\mathbf{E}\left(d_{k} \mid X_{k}, Y_{k}, Z_{k}\right)\right| \leqq C h Y_{k} I_{S_{h}}\left(X_{k}\right) \chi_{æ \geqq k+1 / 2}
$$

holds.

For a half-integer index $k+\frac{1}{2}$, the inequality

$$
\left|\mathbf{E}\left(d_{k+1 / 2} \mid X_{k+1 / 2}, Y_{k+1 / 2}, Z_{k+1 / 2}\right)\right| \leqq C h^{2} Y_{k} \chi_{æ>k+1 / 2}
$$

holds.

TheOREm 4. Algorithm $1^{\prime}$ has weak order of accuracy $O(h)$,

$$
\left|\mathbf{E}\left(\varphi\left(X_{æ}\right) Y_{æ}+Z_{æ}\right)-u\left(x_{0}\right)\right| \leqq C h,
$$

where $C$ is independent of $x_{0}$ and $h$.

Proof. We have

$$
\begin{aligned}
R & :=\mathbf{E}\left(\varphi\left(X_{æ}\right) Y_{æ}+Z_{æ}\right)-u\left(x_{0}\right)=\mathbf{E}\left(u\left(X_{æ}\right) Y_{æ}+Z_{æ}\right)-u\left(x_{0}\right) \\
& =\mathbf{E} \sum_{m=0}^{2 æ-1}\left(u\left(X_{(m+1) / 2}\right) Y_{(m+1) / 2}+Z_{(m+1) / 2}-u\left(X_{m / 2}\right) Y_{m / 2}-Z_{m / 2}\right) \\
& =\sum_{m=0}^{\infty} \mathbf{E} d_{m / 2}=\sum_{k=0}^{\infty} \mathbf{E} d_{k+1 / 2}+\sum_{k=0}^{\infty} \mathbf{E} d_{k} \\
& =\sum_{k=0}^{\infty} \mathbf{E} \mathbf{E}\left(d_{k+1 / 2} \mid X_{k+1 / 2}, Y_{k+1 / 2}, Z_{k+1 / 2}\right)+\sum_{k=0}^{\infty} \mathbf{E} \mathbf{E}\left(d_{k} \mid X_{k}, Y_{k}, Z_{k}\right) .
\end{aligned}
$$

It is obvious that $Y_{k}>0$ and ( since $\left.c(x) \leqq 0\right) Y_{k} \leqq 1, k=0,1, \ldots$. Using Lemmas 4 and 5 , we obtain

$$
\begin{aligned}
|R| & \leqq C h^{2} \sum_{k=0}^{\infty} \mathbf{E}\left(Y_{k} \chi_{æ>k+1 / 2}\right)+C h \sum_{k=0}^{\infty} \mathbf{E}\left(Y_{k} I_{S_{h}}\left(X_{k}\right) \chi_{æ \geqq k+1 / 2}\right) \\
& \leqq C h^{2} \mathbf{E} \rightsquigarrow C h \mathbf{E} \nu_{x_{0}} \leqq C h
\end{aligned}
$$


TABLE 1

Parabolic problem. The results of approximate solution of problem (6.1)-(6.2) according to Algorithm 1 (the upper table) and Algorithm 2 (the lower table) for $\mu \equiv 0, F \equiv 0$. The exact solution $u(0.6,0,0,0) \doteq 0.87797, u(15,0,0,0) \doteq 1.21000$.

\begin{tabular}{|l|l|lr|lr|}
\hline$h$ & $M$ & $\bar{u}(0.6,0,0,0)$ & $\approx \mathbf{E}$ & $\bar{u}(15,0,0,0)$ & $\approx \mathbf{E}$ \\
\hline 0.04 & $1 \cdot 10^{3}$ & $0.7756 \pm 0.0127$ & 7.4 & $1.0337 \pm 0.0180$ & 7.8 \\
\hline 0.01 & $4 \cdot 10^{4}$ & $0.8444 \pm 0.0022$ & 30.6 & $1.1504 \pm 0.0035$ & 32.7 \\
\hline 0.0016 & $1 \cdot 10^{6}$ & $0.8722 \pm 0.0005$ & 194 & $1.1999 \pm 0.0007$ & 207 \\
\hline 0.0001 & $4 \cdot 10^{6}$ & $0.8775 \pm 0.0002$ & 3111 & $1.2093 \pm 0.0004$ & 3325 \\
\hline
\end{tabular}

\begin{tabular}{|l|l|lr|lr|}
\hline$h$ & $M$ & $\bar{u}(0.6,0,0,0)$ & $\approx \mathbf{E}$ & $\bar{u}(15,0,0,0)$ & $\approx \mathbf{E}$ \\
\hline 0.04 & $1 \cdot 10^{3}$ & $0.7127 \pm 0.0115$ & 6.3 & $0.9560 \pm 0.0167$ & 6.3 \\
\hline 0.01 & $4 \cdot 10^{4}$ & $0.7941 \pm 0.0020$ & 27.0 & $1.0719 \pm 0.0030$ & 27.7 \\
\hline 0.0016 & $1 \cdot 10^{6}$ & $0.8440 \pm 0.0004$ & 183 & $1.1526 \pm 0.0007$ & 193 \\
\hline 0.0001 & $4 \cdot 10^{6}$ & $0.8695 \pm 0.0002$ & 3064 & $1.1955 \pm 0.0004$ & 3262 \\
\hline
\end{tabular}

where $C$ is independent of $x_{0}, h$. Theorem 4 is proved.

Remark 2. It is possible to construct algorithms of random walks analogous to Algorithms 2 and 3 for elliptic problem (5.1)-(5.2).

6. Numerical experiment. Consider the Dirichlet problem for the parabolic equation,

$$
\begin{array}{rlrl}
\frac{\partial u}{\partial t}= & \frac{1}{2}\left(1.21-x_{2}^{2}-x_{3}^{2}\right) \frac{\partial^{2} u}{\partial x_{1}^{2}}+\frac{1}{2} \frac{\partial^{2} u}{\partial x_{2}^{2}}+\frac{1}{2} \frac{\partial^{2} u}{\partial x_{3}^{2}} & & \\
& +6\left(1-0.5 e^{-t}\right)\left(x_{1}^{2}\left(1.21-x_{2}^{2}-x_{3}^{2}\right)+x_{2}^{2}\right) & & \\
& +0.5 e^{-t}\left(1.21-x_{1}^{4}-x_{2}^{4}\right), \quad t \in(0, T], \quad & & x \in U_{1}, \\
u(0, x)= & \frac{1}{2}\left(1.21-x_{1}^{4}-x_{2}^{4}\right), & & x \in \bar{U}_{1}, \\
u(t, x)= & \left(1.21-x_{1}^{4}-x_{2}^{4}\right)\left(1-0.5 e^{-t}\right), \quad t \in(0, T], & x \in \partial U_{1},
\end{array}
$$

where $U_{1} \subset \mathbf{R}^{3}$ is a unit ball with center at the origin. This problem has the solution

$$
u(t, x)=\left(1.21-x_{1}^{4}-x_{2}^{4}\right)\left(1-0.5 e^{-t}\right) .
$$

By changing time $t=T-s$ problem (6.1)-(6.2) is rewritten in the form (1.1)-(1.2) which is suitable for the probabilistic approach.

The results of simulation of $u(0.6,0,0,0)$ and $u(15,0,0,0)$ by Algorithms 1 and 2 are given in Table 1 . The values in Table 1 are approximations of $\bar{u}=\mathbf{E}\left(\varphi\left(t_{æ}, X_{æ}\right) \times\right.$ $\left.Y_{æ}+Z_{æ}\right)$, evaluated as

$$
\bar{u} \doteq \frac{1}{M} \sum_{m=1}^{M}\left(\varphi\left(t_{æ}^{(m)}, X_{æ}^{(m)}\right) Y_{æ}^{(m)}+Z_{æ}^{(m)}\right) \pm 2 \sqrt{\frac{\bar{D}_{M}}{M}},
$$

where

$$
\begin{aligned}
\bar{D}_{M}= & \frac{1}{M} \sum_{m=1}^{M}\left[\varphi\left(t_{æ}^{(m)}, X_{æ}^{(m)}\right) Y_{æ}^{(m)}+Z_{æ}^{(m)}\right]^{2} \\
& -\left[\frac{1}{M} \sum_{m=1}^{M}\left(\varphi\left(t_{æ}^{(m)}, X_{æ}^{(m)}\right) Y_{æ}^{(m)}+Z_{æ}^{(m)}\right)\right]^{2} .
\end{aligned}
$$


TABLE 2

Elliptic problem. The results of approximate solution of problem (6.4)-(6.5) according to Algorithm $1^{\prime}$ for $F \equiv 0$. The exact solution $u(0,0,0)=1.21, u(0.5,0,0)=1.1475$.

\begin{tabular}{|l|l|lr|lr|}
\hline$h$ & $M$ & $\bar{u}(0,0,0)$ & $\approx \mathbf{E}$ & $\bar{u}(0.5,0,0)$ & $\approx \mathbf{E}$ \\
\hline 0.04 & $1 \cdot 10^{3}$ & $1.0337 \pm 0.0180$ & 7.8 & $0.9361 \pm 0.0239$ & 5.9 \\
\hline 0.01 & $4 \cdot 10^{4}$ & $1.1504 \pm 0.0035$ & 32.7 & $1.0810 \pm 0.0042$ & 24.3 \\
\hline 0.0016 & $1 \cdot 10^{6}$ & $1.1999 \pm 0.0007$ & 207 & $1.1359 \pm 0.0009$ & 155 \\
\hline 0.0001 & $4 \cdot 10^{6}$ & $1.2093 \pm 0.0004$ & 3325 & $1.1467 \pm 0.0004$ & 2477 \\
\hline
\end{tabular}

Thus, assuming that the sampling variance is sufficiently close to $\bar{D}_{M}, \bar{u}$ belongs to the interval defined in (6.3) with probability 0.95. The Monte Carlo error for values in Table 1 is not greater than errors of numerical integration. As follows from this table, the results of the experiment are in quite good agreement with the theoretical results. Numerical tests of Algorithm 3 also gave results corresponding with the theory.

Consider the Dirichlet problem for the elliptic equation:

$$
\begin{gathered}
\frac{1}{2}\left(1.21-x_{2}^{2}-x_{3}^{2}\right) \frac{\partial^{2} u}{\partial x_{1}^{2}}+\frac{1}{2} \frac{\partial^{2} u}{\partial x_{2}^{2}}+\frac{1}{2} \frac{\partial^{2} u}{\partial x_{3}^{2}} \\
+6 x_{1}^{2}\left(1.21-x_{2}^{2}-x_{3}^{2}\right)+6 x_{2}^{2}=0, \quad x \in U_{1}, \\
\left.u\right|_{\partial U_{1}}=1.21-x_{1}^{4}-x_{2}^{4} .
\end{gathered}
$$

The solution of this problem is

$$
u(x)=1.21-x_{1}^{4}-x_{2}^{4} .
$$

This example was used in [14] to test a method of random walk over touching ellipsoids.

The results of simulation of $u(0,0,0)$ and $u(0.5,0,0)$ by Algorithm $1^{\prime}$ are given in Table 2. The values in Table 2 are approximations of $\bar{u}=\mathbf{E}\left(\varphi\left(X_{\varkappa}\right) Y_{\varkappa}+Z_{\varkappa}\right)$ evaluated by a formula analogous to (6.3). The Monte Carlo error for the values in Table 2 is not greater than the errors of numerical integration. Analyzing the results presented in Table 2, we can conclude that the error of numerical integration is proportional to $C h$ and the mean number of steps $\mathbf{E} \varkappa$ is proportional to $K / h$.

We pay attention to the fact that the method of random walk over touching ellipsoids from [14] is also of the first order of accuracy in $h$ and $\mathbf{E} \varkappa=O(1 / h)$. Comparing the results of tests from [14] with the results in Table 2, we see that the constant $C$ at $h$ in the error of numerical integration is larger for Algorithm $1^{\prime}$ while the constant $K$ at $1 / h$ in the estimate of the mean number of steps $\mathbf{E} \varkappa$ is larger for the method of random walk over touching ellipsoids.

We note (see the introduction) that it is possible to select the function $F$ so that the variance $\bar{D}$ of the random variable $\bar{\eta}=\varphi\left(X_{\varkappa}\right) Y_{\varkappa}+Z_{\varkappa}$ related to the discrete system will decrease. Such a selection allows us to reduce the computational costs. For instance, if we take

$$
F^{\mathrm{T}}=\left(4 x_{1}^{3} \sqrt{1.21-x_{2}^{2}-x_{3}^{2}}, 4 x_{2}^{3}, 0\right),
$$

then it is not difficult to show that the variance $D$ of the random variable $\eta=$ $\varphi\left(X_{x}(\tau)\right) Y_{x, 1}(\tau)+Z_{x, 1,0}(\tau)$ related to the system of SDEs is equal to zero. Since the 
accuracy order of the method is $O(h)$, the variance $\bar{D}$ satisfies the inequality $\bar{D} \leqq C h$, where $C$ is independent of $x, h$. Therefore, the Monte Carlo error is bounded from above by $C \sqrt{h / M}$. In numerical experiments, making use of the function $F$ selected as above leads to a significant decrease of computational costs in comparison with simulations when $F \equiv 0$.

Numerical tests of Algorithm 2' also gave results corresponding with the theory.

\section{REFERENCES}

[1] A. Friedman, Partial Differential Equations of Parabolic Type, Prentice-Hall, Englewood Cliffs, NJ, 1964

[2] E. B. Dynkin, Markov Processes, Springer-Verlag, Berlin, 1965.

[3] I. I. Gikhman And A. V. Skorokhod, Stochastic Differential Equations, Springer-Verlag, Berlin, New York, 1972.

[4] G. N. Milstein, Numerical Integration of Stochastic Differential Equations, Kluwer Academic Publishers, Dordrecht, 1995.

[5] N. J. Newton, Variance reduction for simulated diffusions, SIAM J. Appl. Math., 54 (1994), pp. $1780-1805$.

[6] G. N. Mil'stein, Solution of the first boundary value problem for parabolic equations by integration of stochastic differential equations, Theory Probab. Appl., 40 (1995), pp. 556-563.

[7] C. Costantini, B. Pacchiarotti, and F. Sartoretto, Numerical approximation for functionals of reflecting diffusion processes, SIAM J. Appl. Math., 58 (1998), pp. 73-102.

[8] G. N. Milstein and M. V. Tretyakov, Numerical solution of the Dirichlet problem for nonlinear parabolic equations by a probabilistic approach, IMA J. Numer. Anal., 21 (2001), pp. 887-917.

[9] C. Miranda, Partial Differential Equations of Elliptic Type, Springer-Verlag, Berlin, New York, 1970.

[10] G. N. Milshtein, Application of the numerical integration of stochastic equations to solving boundary-value problems with Neuman's boundary conditions, Theory Probab. Appl., 41 (1996), pp. 170-177.

[11] G. N. MilSTEIN, Weak approximation of a diffusion process in a bounded domain, Stochastics Stochastics Rep., 62 (1997), pp. 147-200.

[12] A. D. Venttsel, A Course in the Theory of Random Processes, 2nd ed., Nauka, Moscow, 1996 (in Russian).

[13] R. Z. KhasminskiI, Stability of Differential Equations under Random Perturbations of Their Parameters, Nauka, Moscow, 1969 (in Russian).

[14] G. N. Milstein And N. F. RyBkina, An algorithm for the method of a random walk on small ellipsoids for the solution of the general Dirichlet problem, Comput. Math. Math. Phys., 33 (1993), pp. 631-647. 